\title{
Superparamagnetic Iron Oxide Nanoparticles: Ampli- fying ROS Stress to Improve Anticancer Drug Efficacy
}

\author{
Gang Huang ${ }^{1 *}$, Huabing Chen ${ }^{1 *}$, Ying Dong ${ }^{1 *}$, Xiuquan Luo ${ }^{1,2}$, Haijun Yu ${ }^{1}$, Zachary Moore ${ }^{1,2}$, Erik A. Bey ${ }^{1,2}$, \\ David A. Boothman ${ }^{1,2}$, Jinming Gao ${ }^{\circledR}$ \\ 1. Departments of Pharmacology, Simmons Comprehensive Cancer Center, University of Texas Southwestern Medical Center at Dallas, \\ Dallas, TX 75390-8807, USA; \\ 2. Radiation Oncology, Simmons Comprehensive Cancer Center, University of Texas Southwestern Medical Center at Dallas, Dallas, TX \\ 75390-8807, USA. \\ *Authors contributed equally to this work.
}

$\triangle$ Corresponding author: Prof. Jinming Gao, David A. Boothman, Simmons Comprehensive Cancer Center, University of Texas Southwestern Medical Center, 5323 Harry Hines Blvd., Dallas, TX 75390. Fax: 214-645-6347; E-mail: jinming.gao@utsouthwestern.edu (J Gao); david.boothman@utsouthwestern.edu (D. Boothman).

(C) Ivyspring International Publisher. This is an open-access article distributed under the terms of the Creative Commons License (http://creativecommons.org/ licenses/by-nc-nd/3.0/). Reproduction is permitted for personal, noncommercial use, provided that the article is in whole, unmodified, and properly cited.

Received: 2012.10.18; Accepted: 2012.12.14; Published: 2013.02.01

\begin{abstract}
Superparamagnetic iron oxide nanoparticles (SPION) are an important and versatile nano- platform with broad biological applications. Despite extensive studies, the biological and pharmacological activities of SPION have not been exploited in therapeutic applications. Recently, $\beta$-lapachone ( $\beta$-lap), a novel anticancer drug, has shown considerable cancer specificity by selectively increasing reactive oxygen species (ROS) stress in cancer cells. In this study, we report that $\mathrm{pH}$-responsive SPION-micelles can synergize with $\beta$-lap for improved cancer therapy. These SPION-micelles selectively release iron ions inside cancer cells, which interact with hydrogen peroxide $\left(\mathrm{H}_{2} \mathrm{O}_{2}\right)$ generated from $\beta$-lap in a tumor-specific, NQOI-dependent manner. Through Fenton reactions, these iron ions escalate the ROS stress in $\beta$-lap-exposed cancer cells, thereby greatly enhancing the therapeutic index of $\beta$-lap. More specifically, a I0-fold increase in ROS stress was detected in $\beta$-lap-exposed cells pretreated with SPION-micelles over those treated with $\beta$-lap alone, which also correlates with significantly increased cell death. Catalase treatment of cells or administration of an iron chelator can block the therapeutic synergy. Our data suggest that incorporation of SPION-micelles with ROS-generating drugs can potentially improve drug efficacy during cancer treatment, thereby provides a synergistic strategy to integrate imaging and therapeutic functions in the development of theranostic nanomedicine.
\end{abstract}

Key words: superparamagnetic iron oxide nanoparticles, $\beta$-lapachone, reactive oxygen species, Fenton reaction, theranostic nanomedicine.

\section{Introduction}

Superparamagnetic iron oxide nanoparticles (SPION or $\mathrm{Fe}_{3} \mathrm{O}_{4}$ ) have been widely used in a variety of biological applications, such as magnetic resonance imaging (MRI), biosensing, magnetic hyperthermia, cell separations, targeted drug delivery, and tissue engineering [1-4]. Several imaging products such as
Feridex IV, Resovist and Lumirem are clinically approved for liver or GI tract imaging [5]. Recently, SPIONs have been incorporated with therapeutic drugs into theranostic nanomedicine platforms for multimodal cancer imaging and image-guided cancer therapy [6, 7]. Our laboratory has previously estab- 
lished SPION-loaded polymeric micelles as ultra-sensitive MRI contrast agents [8-11] or targeted drug delivery systems $[12,13]$. In general, SPIONs are considered inert imaging agents without intended pharmacological functions [14]. In recent years, SPION metabolism has been extensively studied in various biological systems [15-17]. So far, SPIONs have not shown observable short- or long-term toxicities [18-20], after cell uptake and internalization [21-23]. A few studies reported that SPION nanoparticles were degraded and metabolized in endocytic organelles within cells [18, 23], and the resulting iron ions were transported across the endo/lysosomal membranes via the divalent metal transporter-1 (DMT1) into the cytosol [24, 25]. Conceivably, newly formed iron ions may considerably affect the intracellular oxido-reduction reactions and homeostasis of reactive oxygen species (ROS) inside cells. One example is the Fenton reaction (Eqs. 1-2) [26-29]:

$$
\begin{gathered}
\mathrm{Fe}^{2+}+\mathrm{H}_{2} \mathrm{O}_{2} \rightarrow \mathrm{Fe}^{3+}+\mathrm{OH}^{+}+\mathrm{OH}^{-} \\
\mathrm{Fe}^{3+}+\mathrm{H}_{2} \mathrm{O}_{2} \rightarrow \mathrm{Fe}^{2+}+\mathrm{OOH}+\mathrm{H}^{+}
\end{gathered}
$$

where $\mathrm{H}_{2} \mathrm{O}_{2}$ is converted into highly reactive hydroxyl or superoxide radicals catalyzed by $\mathrm{Fe}^{2+} / \mathrm{Fe}^{3+}$ ions. However, there are no reports documenting effects of SPION $/ \mathrm{Fe}^{2+} / \mathrm{Fe}^{3+}$ on drug efficacy inside cancer cells.

Recently, exploiting increased ROS levels and altered redox status in cancer cells has become a new therapeutic strategy to improve cancer selectivity over normal cells [30]. Moderate increases in ROS may confer cancer cells advantages of faster genetic mutations, higher cell proliferation and elevated differentiation rates. However, cancer cells are also more susceptible to oxidative insults compared to normal cells. Additional ROS stress induced by exogenous agents can overwhelm the relatively low antioxidant capacity and disrupt the redox homeostasis inside cancer cells, which can lead to selective tumor cell toxicity. A variety of therapeutic drugs that either generate ROS or inhibit antioxidant enzymes have been developed for cancer treatment [30-32]. For example, motexafin gadolinium, which can selectively accumulate in tumor cells and induce intracellular superoxide formation for cytotoxicity, has been evaluated in phase III trials for cancer therapy [33]. In another example, mangafodipir acts as antioxidant in normal cells, but increases $\mathrm{H}_{2} \mathrm{O}_{2}$ levels in cancer cells, showing synergy in combination with chemotherapy among liver cancer patients in phase II studies [34].

In the past 10 years, our labs have studied an exogenous ROS-generating agent, $\beta$-lapachone
( $\beta$-lap), for cancer chemotherapy [35, 36]. Catalysed by $\mathrm{NAD}(\mathrm{P}) \mathrm{H}$ : quinone oxidoreductase 1 (NQO1) that is specifically overexpressed in breast, prostate, lung, and pancreatic cancer cells, $\beta$-lap can undergo futile redox cycles to generate elevated superoxide $\left(\mathrm{O}_{2}{ }^{-}\right)$and $\mathrm{H}_{2} \mathrm{O}_{2}$, causing massive ROS levels and poly(ADP-ribose) polymerase 1 (PARP1) hyperactivation-mediated programmed necrosis (Scheme 1/Figure S1). Combination of iron ions with $\beta$-lap can further produce highly reactive hydroxyl radicals through Fenton reactions, increasing the antitumor activity of $\beta$-lap, lowering its effective dose. In most cells, intracellular iron ions are generally bound in specific proteins and only trace amount of free ions are available for Fenton reactions [37]. Herein, we investigate a nanoplatform composed of $\mathrm{pH}$-responsive SPION-micelles to investigate its therapeutic synergy with $\beta$-lap. We hypothesize that once taken up by the cancer cells, SPION-micelles can act as an intracellular iron donor, degraded in acidic organelles such as endosomes $(\mathrm{pH}$ 5.5-6.3) or lysosomes ( $\mathrm{pH}$ 5.0-5.5) [38], with $\mathrm{pH}$-dependent release of iron ions [39] (Scheme 1/Figure S1). In the presence of these iron ions, hydrogen peroxide generated from NQO1-specific $\beta$-lap futile redox cycling will be transformed into highly reactive hydroxyl radicals, and increase ROS stress in the cancer cells. Integration of SPION-micelles with ROS-based anticancer drugs can potentially improve drug targeting specificity and therapeutic efficacy in cancer therapy.

\section{Materials and Methods}

Preparation and Characterization of SPION-micelles. SPION (5.8 $\mathrm{nm})$ was first synthesized following a published procedure [40]. The $\mathrm{pH}$-sensitive amphiphilic copolymer, poly(ethylene glycol)-b-poly(2-(2-diisopropylamino) ethyl methacrylate $\left(\mathrm{PEG}_{114}-b-\mathrm{PDPA}_{120}\right)$, was synthesized by atomic transfer radical polymerization (ATRP) [41]. For the preparation of $\mathrm{pH}$-responsive SPION micelles, $8.0 \mathrm{mg}$ PEG- $b$-PDPA and $2.0 \mathrm{mg}$ SPIONs were first dissolved in $1.0 \mathrm{~mL}$ tetrahydrofuran (THF) in a glass vial. Then the solution was added to purified water $(3.0 \mathrm{~mL})$ under vigorous ultrasonic agitation using a Type 60 Sonic Dismembrator (Fisher Scientific). The vial was then open to air overnight, allowing evaporation of THF and formation of micelles. The residual THF was completely removed using centrifugation dialysis (MW cutoff $100 \mathrm{kD}$, Millipore, MA) (3,000 rpm, 30 min, 3 times). Finally, SPION micelles were filtered through $0.22 \mu \mathrm{m}$ filter and stored at $4{ }^{\circ} \mathrm{C}$. 


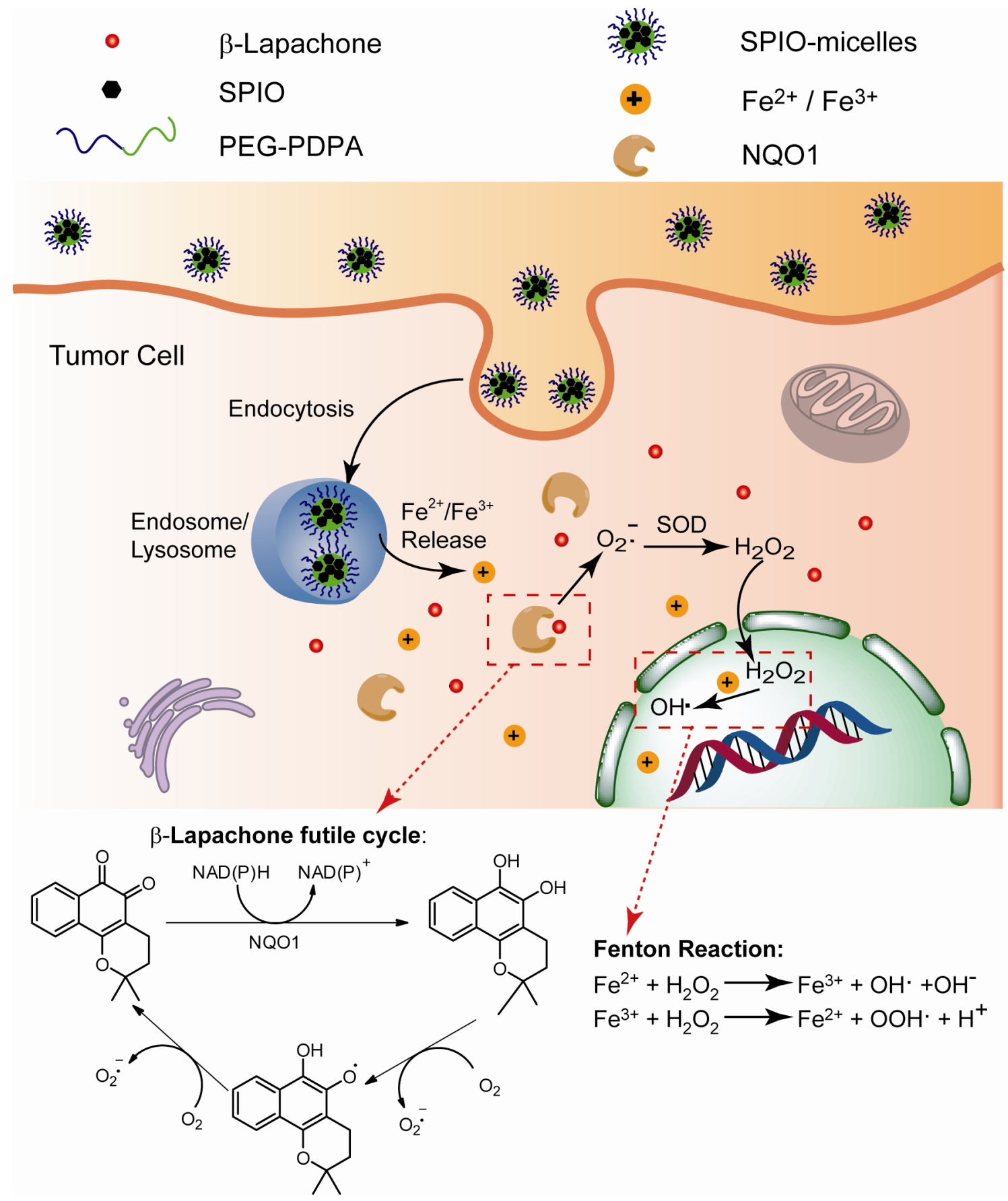

Figure SI (Scheme I). Schematic illustration of intracellular distribution of $\mathrm{pH}$-sensitive SPION-micelles, iron ion release, subsequent Fenton reactions with $\mathrm{NQO}$-dependent $\mathrm{H}_{2} \mathrm{O}_{2}$ generated from $\beta$-lapachone ( $\beta$-lap) futile redox cycle to amplify ROS stress levels for improved antitumor efficacy. NQOI - NAD(P)H: quinone oxidoreductase I; SOD - superoxide dismutase.

Transmission electron microscopy (TEM) was performed to examine the particle size and morphology using a JEOL 1200 EX II instrument (Tokyo, Japan). Each sample was first diluted using water and then placed on a glow discharged copper grid and allowed to adsorb for 45 seconds. The excess solution was removed by blotting the grid against filter paper and then the grid was stained for 30 seconds using $2 \%$ phosphotungstic acid. After the excess solution was removed, the grid was directly dried in the air before TEM analysis. Dynamic light scattering (DLS) was used to measure the size of SPION micelles in solution. The hydrodynamic diameters of SPION micelles were characterized by a Zetasizer (Malvern NanoZS model).

Release of Iron Ions from SPION-micelles. 
Release of iron ions from SPION-micelles was measured using a dialysis setup (MW cut-off $3000 \mathrm{Da}$ ) in an oscillator shaker. SPION-micelles $(1.0 \mathrm{mM}, 1.0 \mathrm{~mL})$ were placed in a dialysis tube and the tube was placed into $20 \mathrm{~mL}$ buffers as the release media at $\mathrm{pH} 7.4,6.2$ and $5.0\left(\mathrm{~T}=37^{\circ} \mathrm{C}\right)$. At selected time points, buffered solutions outside the dialysis tube $(1.0 \mathrm{~mL}$ each) were taken with the replacement of an equal volume of new release medium. The concentration of iron ions was measured by atom absorption spectrometer (Spectra AA, Varian).

Relative Cell Survival Assays. A549 non-small cell lung carcinoma (NSCLC) cells were grown in Dulbecco's minimal essential medium (DMEM) with $5 \%$ fetal bovine serum (FBS). Cells were cultured at 37 ${ }^{\circ} \mathrm{C}$ in a $5 \% \mathrm{CO}_{2}-95 \%$ air humidified atmosphere and were free from mycoplasma contamination. Briefly, cells were treated with SPION-micelles and $\beta$-lap at indicated concentrations with or without $48 \mathrm{~h}$ pretreatment of SPION-micelles. When indicated, deferoxamine $(5 \mathrm{mM})$ or Catalase $(1000 \mathrm{U})$ pre- and co-treatments were also studied. Drug-free media were then added and cells allowed to grow for another 7 days or until control cells reached $\sim 95 \%$ confluence. DNA contents of samples were determined by Hoechst 33258 dye staining and fluorescence detection using a plate reader (Perkin-Elmer, Boston, MA) [42]. Results were reported as means \pm standard error (SE) from sextuplet repeats. Statistical analysis was performed in all the experiments. The $p$ values were calculated using the Students' two-tailed $t$ test for paired comparisons, and $p<0.05$ is considered statistically significant.

Confocal Laser Scanning Microscopy. SPION micelles from rhodamine-conjugated PEG- $b$-PDPA copolymer (iron concentration $=0.14 \mathrm{mM}$ ) were incubated with A549 cells for $4 \mathrm{~h}$, followed by treatment with LysoTracker Green DND26 (50 nM) for 5 minutes. Live cell imaging was performed on a confocal laser scanning microscope (Nikon TE2000-E model). The excitation/emission wavelengths were set at 488/510 $\mathrm{nm}$ for LysoTracker Green and 543/590 nm for rhodamine, respectively.

Western Immunoblotting. Whole-cell extracts were prepared, and Western blots were developed using SuperSignal West Pico Chemiluminescent substrate (Thermo Scientific) and exposed using autoradiography film (Denville Scientific, Inc.). PAR antibodies (BD Pharmagin ${ }^{\mathrm{TM}}$ ) were used at 1:2000 dilutions.

ROS Formation. ROS formation was monitored by fluorescence microscopy using the superoxide indicator dihydroethidium (DHE) staining. Briefly, cells were seeded on a 35-mm glass cover slip and allowed to attach overnight followed by $48 \mathrm{~h}$ pre-incubation of SPION-micelles $(0.14 \mathrm{mM})$ when described. Cells were then loaded with $10 \mu \mathrm{M}$ of DHE in phosphate buffered saline (PBS) for $30 \mathrm{~min}$ at $37^{\circ} \mathrm{C}$, washed twice with PBS, and co-treated with $3 \mu \mathrm{M} \beta$-lap and $0.14 \mathrm{mM}$ SPION-micelles. At indicated time points, cells were fixed in $10 \%$ formalin for $30 \mathrm{~min}$ at room temperature, then washed twice in PBS and mounted in Vectashield (with DAPI, Vector Laboratories, Inc., Burlingame, CA). Images were taken using a Leica DM5500 fluorescence microscope (Buffalo Grove, IL). Quantitative data analysis was performed using NIH Image J software, where 50 random cells were chosen and the fluorescence intensities were measured and compared with the fluorescence intensities of untreated control cells to quantify the ROS level (treated over control). Each data point represented the mean \pm SE of 50 cells and data were representative of experiments performed in triplicate.

\section{Results}

Characterization of SPION-loaded micelles. Figure $1 \mathrm{~A}$ shows the transmission electron microscopy (TEM) image of a representative SPION-micelle sample, where a cluster of SPIONs was encapsulated inside the hydrophobic core of poly(ethylene glycol)-b-poly(2-(2-diisopropylamino) ethyl methacrylate (PEG- $b$-PDPA) micelles. SPIONs alone had a diameter of $5.8 \pm 1.0 \mathrm{~nm}$ (Fig. $1 \mathrm{~A}$ inset). SPION/PEG- $b$-PDPA micelles had an average hydrodynamic diameter of $65 \pm 5 \mathrm{~nm}$, which was significantly larger than blank PEG- $b$-PDPA micelles (32 \pm 3 $\mathrm{nm}$, data not shown).

Release profile of iron ions from SPION-micelles at different $\mathrm{pH}$ values. In order to evaluate the release behavior of iron ions from SPION-micelles in an acidic environment, we measured the kinetics of iron ion release from SPION-micelles at different $\mathrm{pH}$. At $\mathrm{pH} 5.0$ and 6.2, SPION-micelles released $60 \pm 2 \%$ and $11 \pm 1 \%$ of total iron ions after $72 \mathrm{~h}$, respectively (Fig. 1B). In contrast, release of iron ions from micelles was not observable at $\mathrm{pH}$ 7.4. At $\mathrm{pH} 7.4$, the neutral PDPA segments encapsulated SPION in the hydrophobic core of the micelles, making SPIONs inaccessible to water. While at lower $\mathrm{pH}$ (i.e. $\mathrm{pH}=6.2$ or lower), the tertiary amines on the PDPA segments became protonated and positively charged, leading to micelle dissociation and facile release of iron ions.

Intracellular uptake and lysosomal distribution of SPION-micelles in A549 NSCLC cells. To track the intracellular distribution of SPION-micelles, we synthesized rhodamine-conjugated PEG- $b$-PDPA to produce $\mathrm{pH}$-activatable fluorescent SPION-micelles 
[41]. These micelles have ultra-sensitive $\mathrm{pH}$ response at 6.2, and their fluorescence can be selectively activated in endocytic compartments such as endosomes or lysosomes [41]. LysoTracker Green DND-26 was selected as a green marker for labeling acidic late endosomes and lysosomes. Confocal imaging was used to monitor the intracellular distribution of micelles in A549 lung cancer cells. Majority of the SPION-micelles $(>80 \%)$ were localized in late endo- somes and lysosomes after $4 \mathrm{hr}$ incubation, as evidenced by co-localization of red fluorescence with LysoTracker Green (Fig. 1C). Residence of SPION-micelles in the acidic microenvironment resulted in subsequent release of iron ions $[39,43]$. The iron ions can be transported through the membrane and diffuse into cytosol, which acts as a reservoir of free iron ions for increasing ROS stress [21,39].
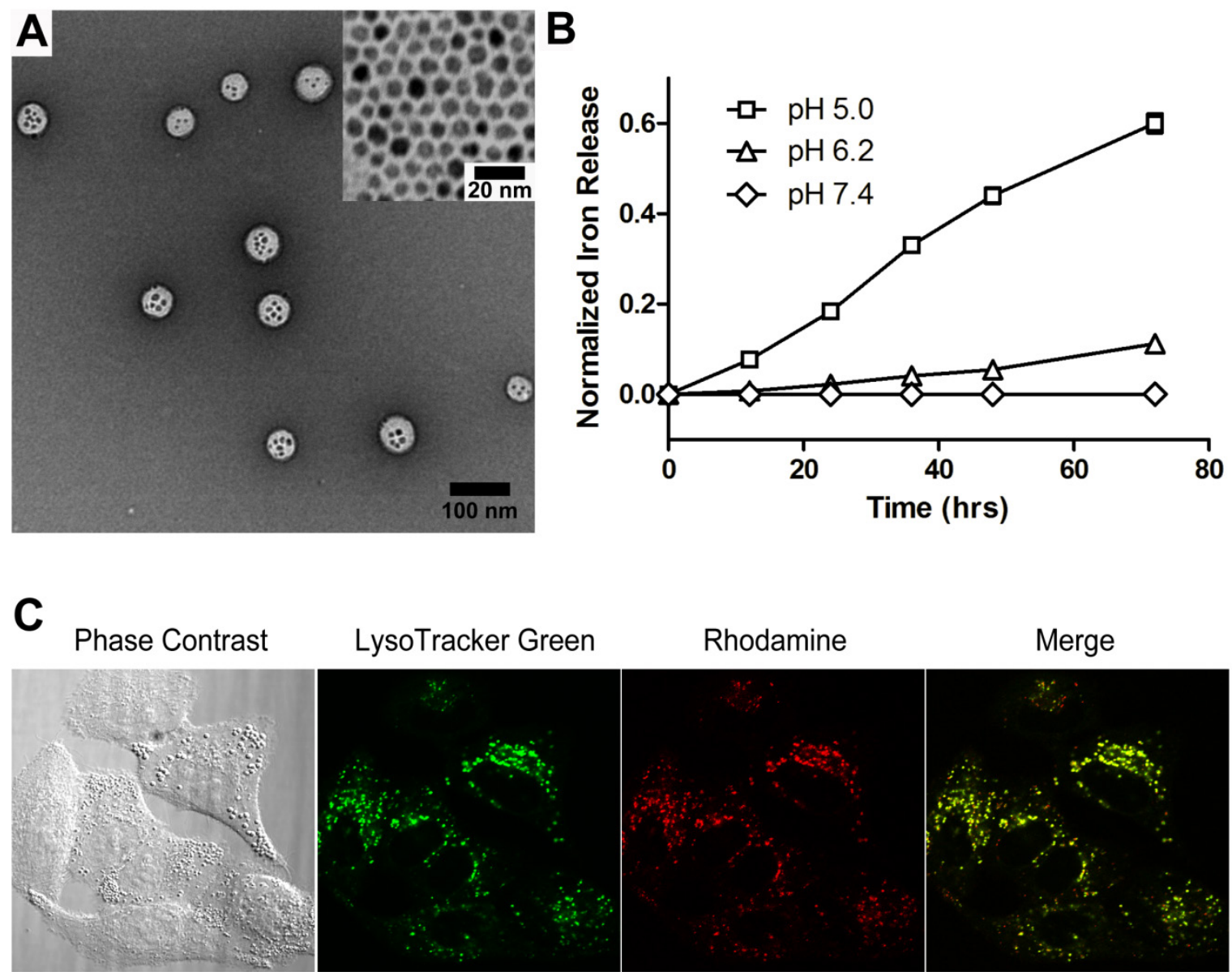

Figure I. (A) Transmission electron microscopy (TEM) image of a representative SPION-micelle sample (inset TEM image shows $5.8 \pm$ $1.0 \mathrm{~nm}$ SPIO nanoparticles). (B) Release profiles of iron ions from SPION-micelles at various pH values. (C) Confocal laser scanning microscopy of intracellular distribution of rhodamine-labelled SPION-micelles in A549 NSCLC cells stained with LysoTracker green after $4 \mathrm{~h}$ incubation. Dissociation of SPION-micelles in acidic organelles resulted in activation of rhodamine signal (red), which co-localized with lysosomes (green).

Enhanced ROS stress in B-lap-exposed A549 NSCLC cells pretreated with SPION-micelles. To evaluate if released iron ions can interact with $\mathrm{H}_{2} \mathrm{O}_{2}$ to increase ROS stress in cancer cells, we used dihydroethidium (DHE) staining to detect ROS levels in $\beta$-lap-exposed A549 cells under different conditions (Fig. 2A-B). In this assay, DHE is oxidized by hydroxyl or superoxide radicals yielding 2-hydroxyethidium, which intercalates DNA and emits bright fluorescence signals inside cell nuclei. Because it took $48 \mathrm{hrs}$ for SPION-micelles to release $40 \%$ of total iron ions at pH 5.0 (Fig. 1B), we used $48 \mathrm{hr}$ pretreatment of SPION-micelles in all of the following experiments. For control group, cells treated with SPION-micelles only didn't show any fluorescence inside the nuclei (data not shown). A549 cells without SPION pretreatment had very weak fluorescence inside nuclei after exposure to $\beta$-lap $(3 \mu \mathrm{M})$ at early time 
points, indicating modest ROS generation from $\beta$-lap redox cycle (Fig. 2A). After 60 mins, ROS stress was still kept at a very low level due to the limited ROS formation. In contrast, cells pretreated with SPION-micelles at $0.14 \mathrm{mM}$ showed a similar low fluorescence at 10 mins, but the fluorescence intensity increased dramatically at 20 and 60 mins of $\beta$-lap incubation, compared to those cells without pretreatment. Quantitative image analysis showed that 20 mins after $\beta$-lap treatment, cells pretreated with 0.14 mM SPION-micelles had 12-fold higher fluorescence intensity compared to cells without SPION-micelle

A

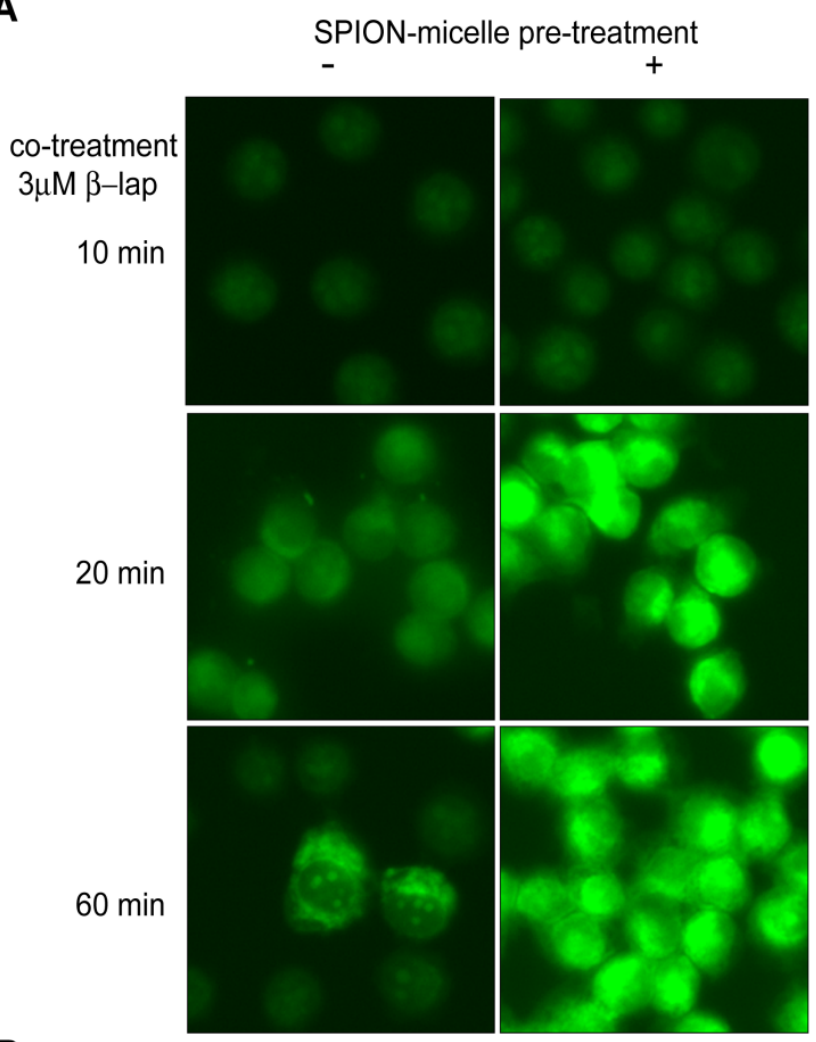

B

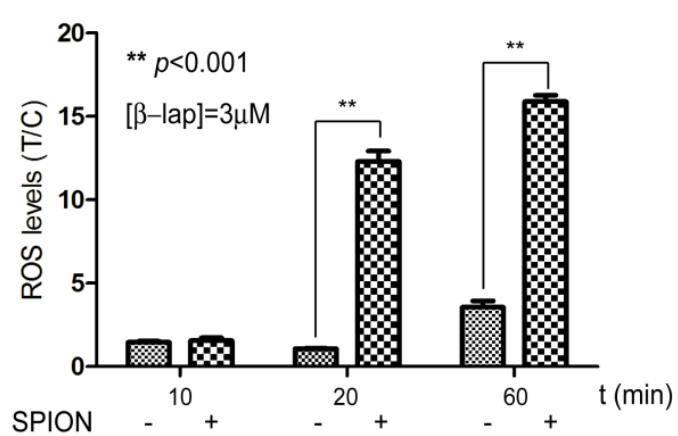

C

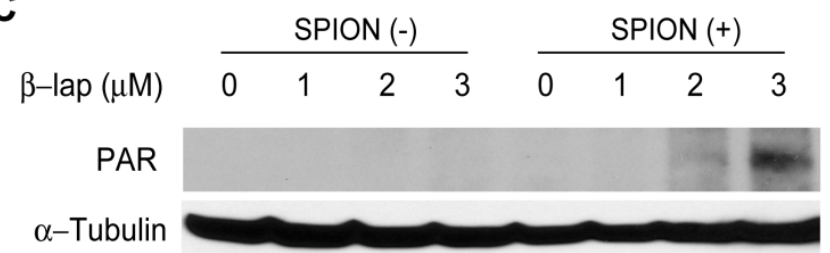

pretreatment $(p<0.001)$, indicating an over 10 -fold increase in ROS level. After 60 mins of $\beta$-lap treatment, the fluorescence intensity increased 16-fold for cells pretreated with SPION-micelles, a 5-fold enhancement over cells without pretreatment $(p<0.001)$ (Fig. $2 B)$. In addition, lethal dose of $\beta$-lap is known to induce programmed necrosis involving PARP1 hyperactivation as a result of released $\mathrm{Ca}^{2+}$ and extensive DNA single strand breaks (SSB) and base damages [36, 44]. However, $\beta$-lap alone at sublethal concentrations (i.e., $3 \mu \mathrm{M}$ ) didn't cause observable PARP1 hyperactivation as measured by PAR formation within $15 \mathrm{~min}$ after treatment (Fig. 2C). In comparison, pretreatment with SPION-micelles dramatically elevated ROS levels in the cells at sublethal dose of $\beta$-lap, caused significantly increased levels of SSB and PARP1 hyperactivation (Fig. 2C).

Figure 2. (A) Fluorescent images of $A 549$ cells treated with SPION-micelles and $\beta$-lap under different conditions using DHE staining for hydroxyl and superoxide radical detection. (B) Quantification of fluorescence intensity changes reflecting ROS levels in A549 NSCLC cells pretreated with or without SPION-micelles followed by exposure to $\beta$-lap. Fluorescent intensity of each image was quantified using Imagej; values are means \pm SE from at least 50 cells in triplicate experiments. (C) Western blot analyses of PAR formation in A549 cancer cells treated with different $\beta$-lap concentrations, with or without SPION-micelle pretreatment $(0.07 \mathrm{mM})$.

Synergy of SPION-micelles and $\beta$-lap in treating A549 NSCLC cells. To further evaluate if SPION-micelles can improve the cytotoxicity of $\beta$-lap by amplifying ROS stress in vitro, we monitored long-term survival changes using a 7-day DNA assay after treating A549 NSCLC cells with various SPION-micelles, with or without $\beta$-lap treatment conditions. SPION-micelles at $0.14 \mathrm{mM}$ iron concentration, as well as $\beta$-lap at sublethal concentrations $(1-3 \mu \mathrm{M})$, did not result in significant cytotoxicity (Fig. 3A) [36]. However, pretreatment of A549 cells with $0.14 \mathrm{mM}$ SPION-micelles followed by the sublethal $\beta$-lap concentrations showed significantly increased lethality compared to $\beta$-lap-exposed cells without SPION-micelle pretreatment. For example, exposure of $3 \mu \mathrm{M} \quad \beta$-lap-treated A549 cells with SPION-micelle pretreatment had a survival rate of only $10 \pm 6 \%$, much lower than in cells without SPION-micelle pretreatment, which was $72 \pm 1 \%$ $(p<0.001)$. To further characterize synergy, survival 
levels were evaluated at different iron concentrations, while maintaining a sublethal $3 \mu \mathrm{M} \beta$-lap dose (Fig. $3 \mathrm{~B})$. Because SPION-micelles failed to induce significant ROS stress, even at high concentrations (up to 0.5 $\mathrm{mM})$, no cytotoxicity was observed (data not shown). In contrast, pretreatment of SPION-micelles at different concentrations significantly increased $\beta$-lap cytotoxicity (Fig. 3B). Lethality decreased from 61 $\pm 1.7 \%$ to $9.7 \pm 5.6 \%$, when the iron concentration increased to $0.07 \mathrm{mM}$. Importantly, the synergy in cytotoxic effects of SPION-micelle $+\beta$-lap treatments were NQO1 dependent, since a specific NQO1 inhibitor, dicoumarol (Dic, $40 \mu \mathrm{M}$ ) completely abrogated lethality (Fig. 3B).
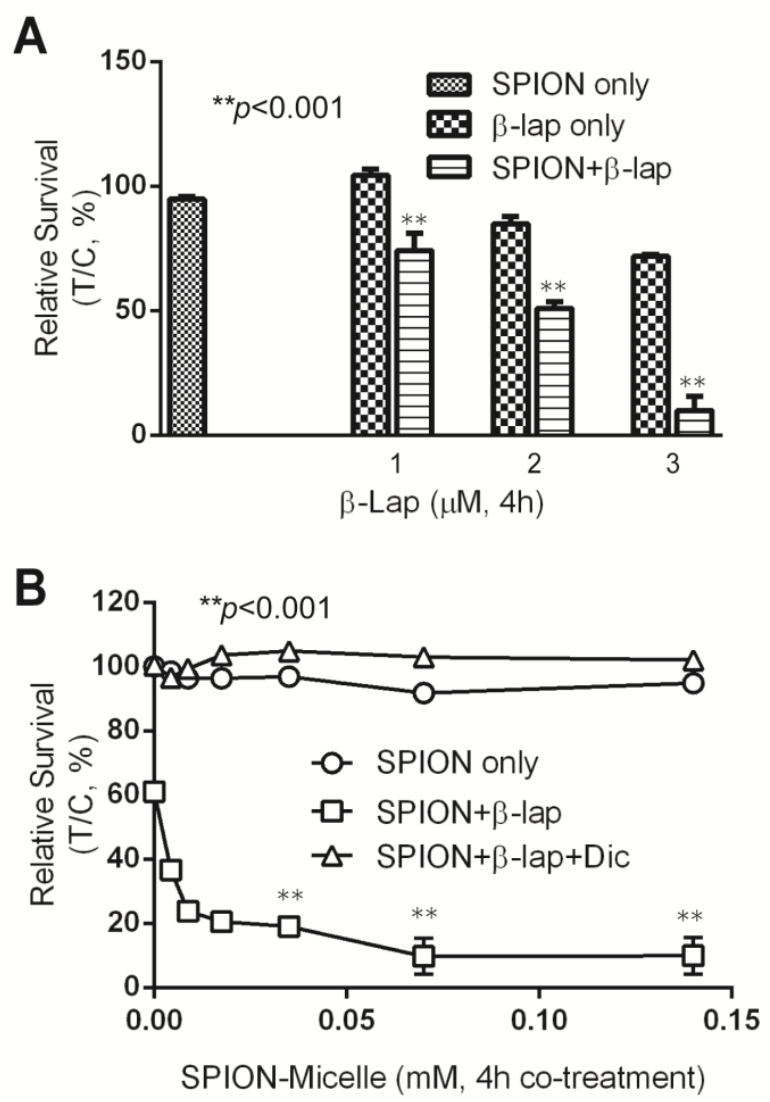

Figure 3. (A) Cell survival study of $A 549$ cells with or without pretreatment of $0.14 \mathrm{mM}$ SPION-micelles for $48 \mathrm{hrs}$ followed by treatment with various $\beta$-lap concentrations. (B) Survival study of A549 cells pretreated with $0.14 \mathrm{mM}$ SPION-micelles for $48 \mathrm{hrs}$, followed by $3 \mu \mathrm{M} \beta$-lap for 4 hrs with or without dicoumarol.

Catalase and an iron chelator protect cells from ROS cytotoxicity. To evaluate whether $\mathrm{H}_{2} \mathrm{O}_{2}$ is the primary reactive oxygen intermediate for the synergistic efficacy caused by SPION-micelles and $\beta$-lap, Catalase, the intracellular $\mathrm{H}_{2} \mathrm{O}_{2}$ scavenger, was exog- enously administered to cells during $\beta$-lap addition to interfere with ROS stress formation. Without Catalase, pre-treatment of SPION-micelles led to significant cell death at sublethal concentration of $\beta$-lap (Fig. $4 \mathrm{~A})$. When Catalase (1000 U) was added into the culture media, it blocked the synergistic effects of SPION-micelles. Survival increased from $24 \pm 2 \%$ to $72 \pm 6 \%$ for SPION-micelles delivering an iron concentration at $0.07 \mathrm{mM}(p<0.001)$. These data suggest that $\mathrm{H}_{2} \mathrm{O}_{2}$ generated by NQO1-dependent futile cycling of $\beta$-lap was detoxified by Catalase forming water and oxygen, which blocks its reaction with iron ions to form more reactive hydroxyl radicals [45].

A

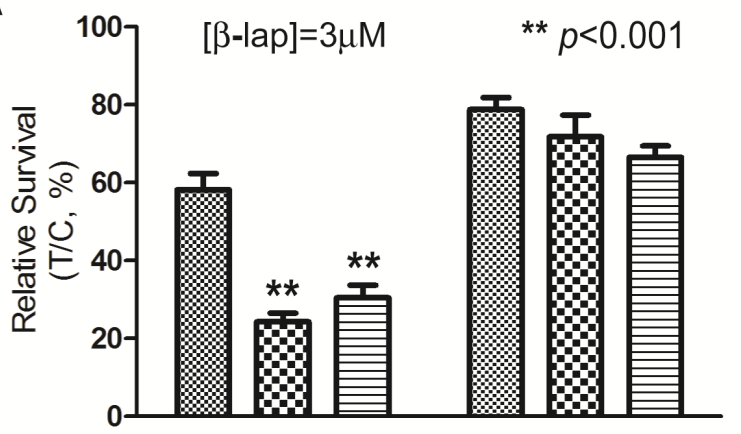

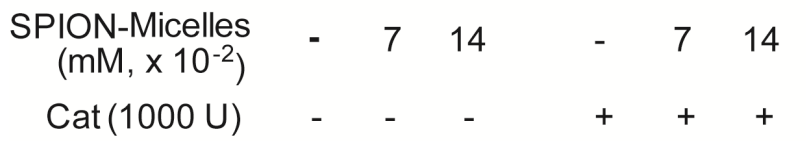

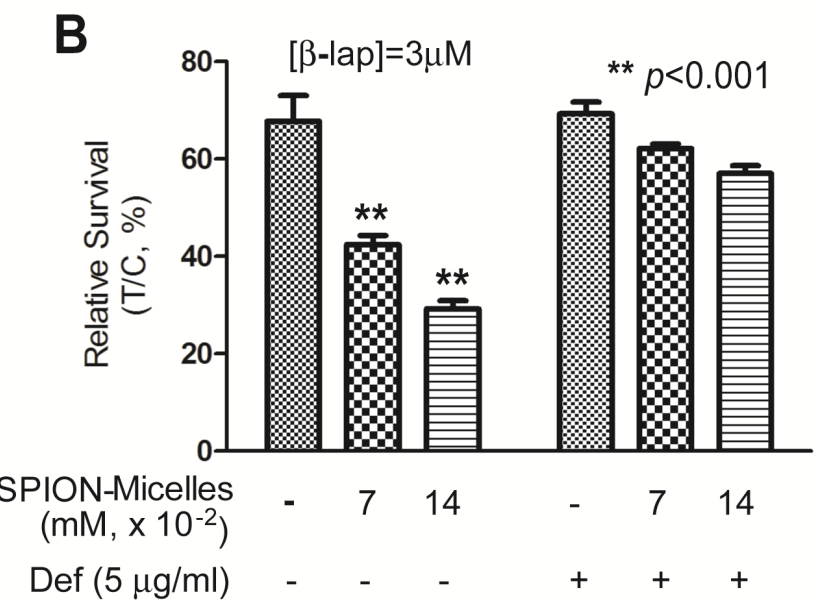

Figure 4. (A) Cytotoxicity of A549 NSCLC cells pretreated with SPION-micelles at $0.07 \mathrm{mM}$ or $0.14 \mathrm{mM}$ for $48 \mathrm{hrs}$ followed by exposure to $3.0 \mu \mathrm{M} \beta$-lap for $4 \mathrm{hrs}$ with or without the $\mathrm{H}_{2} \mathrm{O}_{2}$ scavenger, catalase (1000 U). (B) Survival study of A549 cells pretreated with $0.07 \mathrm{mM}$ or $0.14 \mathrm{mM}$ SPION-micelles for $48 \mathrm{hrs}$, with or without the iron chelator, deferoxamine (Def, $5 \mu \mathrm{g} / \mathrm{mL}$ ), with or without $3 \mu \mathrm{M} \beta$-lap for $4 \mathrm{hrs}$. Data presented are means \pm $\mathrm{SE}$ from experiments performed in triplicate. 
To further confirm that the released iron ions from SPION-micelles are indeed responsible for the noted synergistic cytotoxicity, we used deferoxamine, an FDA-approved iron-chelating agent that binds free iron ions in the body [46, 47], to prevent Fenton reactions with $\mathrm{H}_{2} \mathrm{O}_{2}$. A549 cells were pretreated with SPION-micelles $(0.07 \mathrm{mM}$ and $0.14 \mathrm{mM}$ iron, respectively) with or without $5.0 \mu \mathrm{g} / \mathrm{mL}$ deferoxamine, followed by a $4 \mathrm{hr}$ treatment with sublethal $3.0 \mu \mathrm{M} \beta$-lap (Fig. 4B). Survival of cells exposed to $0.07 \mathrm{mM}$ iron from SPION-micelles increased from $42 \pm 2 \%$ to $62 \pm 1 \%$ when deferoxamine was added. A similar trend was also observed at $0.14 \mathrm{mM}$ iron delivered by SPION-micelles, where survival increased from $29 \pm 2 \%$ to $57 \pm 2 \% \quad(p<0.001)$. These data suggest that deferoxamine may bind to released iron ions from SPION-micelles and formed Fe-chelates in cells. Since deferoxamine does not interfere with NQO1 activity, the futile cycling of $\beta$-lap by NQO1, or chelate $\mathrm{Ca}^{2+}$ [44], these data suggest that the specific presence of the iron ions from SPION-micelles as well as $\mathrm{H}_{2} \mathrm{O}_{2}$ from $\beta$-lap are two prerequisites for achieving the synergistic efficacy in cancer cells.

\section{Discussion}

For the past two decades, SPIONs have been clinically used as MRI contrast probes in cancer diagnosis [5-7]. Recent efforts in SPION research have been devoted to developing structurally well-defined SPIONs for improved magnetization, or incorporating more versatile surface groups for cancer-specific imaging applications [19]. On the other hand, the biological and pharmacological functions of these iron-rich nanoparticles are rarely exploited. In fact, the exogenous supply of iron ions and their delivery into cells are essential for cell metabolism, growth and proliferation [25]. Under aerobic conditions, free iron ions can catalyze the propagation of ROS and generation of highly reactive radicals through Fenton reactions. This characteristic provides a new strategy to utilize SPION for ROS amplification in combination with antitumor agents for improved cancer therapy.

It is well known that cancer cells have heightened level of oxidative stress due to the fast cell proliferation and metabolism compared to normal cells. Although the increased ROS stress confers mutation advantages in some cases, it also makes the cancer cells more susceptible for ROS-induced toxicity [30]. Therefore, manipulating ROS levels by redox modulation from exogenous agents may provide a useful strategy to selectively kill cancer cells without causing significant toxicity to normal cells. $\beta$-Lap is a novel anticancer agent with selectivity against cancer cells that overexpress two-electron oxidoreductase NQO1.
NQO1 induces a futile cycling of $\beta$-lap that exhausts $\mathrm{NAD}(\mathrm{P}) \mathrm{H}$ reducing equivalents in cancer cells, leading to a dramatic increase in ROS levels that causes specific DNA base damage and DNA SSBs. Massive levels of SSBs that result from $\beta$-lap exposures hyperactivate PARP1, leading to a unique programmed cell death mechanism (Fig. 5). Since a threshold level of SSB formation is required to hyperactivate PARP1, synergy can result between $\beta$-lap and agents that cause DNA lesions, including ionizing radiation [48]. We reasoned, and our results support the hypothesis, that co-administering SPION-micelles as a source of intracellular iron ions could augment ROS levels generated by NQO1 futile cycling of otherwise sublethal doses of $\beta$-lap, to synergistically meet the SSB DNA lesion threshold required for PARP1 hyperactivation (Scheme 1/Figure S1). Once PARP1 hyperactivation is met, cell death without resistance is ensured [49].

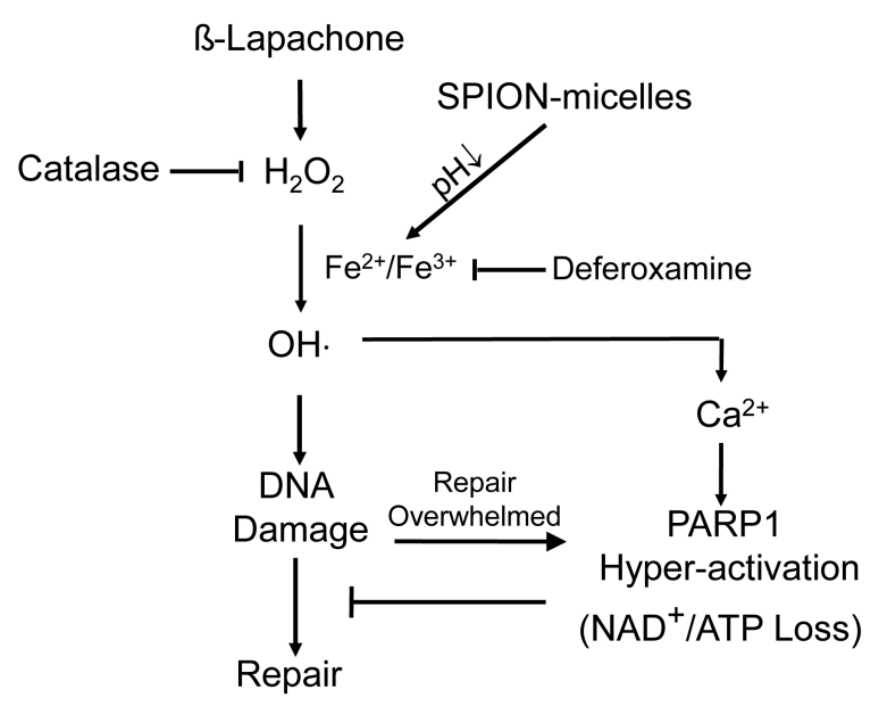

Figure 5. Enhanced cell death mechanism at sublethal dose of $\beta$-lap as induced by SPION-micelles. Production of hydroxyl radicals are dramatically elevated in cancer cells exposed to $\beta$-lap and SPION-micelles, which results in DNA damage, $\mathrm{Ca}^{2+}$ release, and eventually PARP-I hyperactivation. Catalase and deferoxamine can prevent the synergized lethality by consuming the $\mathrm{H}_{2} \mathrm{O}_{2}$ or chelating iron ions, respectively.

The objective of this study was to investigate $\mathrm{pH}$-responsive SPION-micelles and their synergy with $\beta$-lap for improved drug efficacy and therapeutic index. SPION-micelles offer a continuous $\mathrm{Fe}^{2+} / \mathrm{Fe}^{3+}$ release in acidic organelles, such as late endosomes and lysosomes, in cancer cells. A fraction of these iron ions can enter the cytosol and eventually penetrate into the nucleus, where in each location they can escalate ROS levels through Fenton reactions with $\mathrm{H}_{2} \mathrm{O}_{2}$ 
in cancer cells as generated by $\beta$-lap (Scheme 1/Figure S1). This ROS generation/escalation mechanism is different from reports by other groups, where reactive hydroxyl radicals were directly produced at SPION surface $[29,50]$. The latter mechanism may not be adequate in the generation of hydroxyl radicals inside cell nucleus for DNA damage due to the trapping of SPION inside endosomes/lysosomes. In contrast, we anticipate that the released $\mathrm{Fe}^{2+} / \mathrm{Fe}^{3+}$ as well as $\mathrm{H}_{2} \mathrm{O}_{2}$ can be more freely to diffuse into the nucleus to generate elevated ROS stress for efficient DNA damage. This hypothesis is in agreement with the deferoxamine experiment where the iron chelator can efficiently block the increased cytotoxicity of $\beta$-lap due to pretreatment of SPION-micelles, whereas it should have little effect on the hydroxyl radical formation at SPION surface.

In cancer therapy, strategies to combine two anticancer drugs or combine drugs with radiation are normally applied to improve therapeutic outcome. However, those methods may not always lead to a selective killing of the cancer cells. Under persistent intrinsic oxidative stress, ROS scavenging systems can be upregulated and cells may become well-adapted to such stress, acquiring resistance. In addition, ROS stress levels caused by indiscriminate cytotoxic antitumor exposure of normal cells may also increase lethality, leading to major normal tissue complications and concerns of drug safety. Our data suggest that administration of SPION-micelles followed by $\beta$-lap may provide a safer and more efficacious treatment regimen that specifically targets cancer cells that overexpress NQO1. In the absence of $\mathrm{H}_{2} \mathrm{O}_{2}$, SPION-micelle itself did not appear to induce ROS stress. However, when $\beta$-lap was administered and significant NQO1-dependent $\mathrm{H}_{2} \mathrm{O}_{2}$ levels increased, released $\mathrm{Fe}^{2+} / \mathrm{Fe}^{3+}$ from SPION-micelles amplified ROS levels, resulting in cell-death threshold levels of DNA damage. Since, in general, normal cells lack NQO1 expression, the presence of iron ions together with $\beta$-lap treatment would not result in cytotoxic effects since $\mathrm{H}_{2} \mathrm{O}_{2}$ is not generated without NQO1 futile cycling.

Although we mainly investigated the biological activity of SPION-micelles in combination with $\beta$-lap in vitro, the imaging functions of SPION can still be utilized to provide vital information on tumor responses and nanoparticle delivery in vivo. By functionalization of SPION-micelles with specific cancer targets, these nanoparticles can be specifically delivered to the tumor site, providing early feedback of therapeutic efficacy by SPION and $\beta$-lap, monitoring real-time tumor responses. Nevertheless, current system still needs to address several limitations before preclinical animal studies or clinical translations. First, iron release kinetics from the current SPIO-micelles were still relatively slow (e.g. $20 \%$ after $24 \mathrm{hrs}$ at $\mathrm{pH}$ 5.0), which requires the pretreatment of cancer cells with SPIO-micelles before drug exposure. This prevented the incorporation of both drug and SPIONs into a single theranostic system to achieve similar pharmacokinetics and tumor distribution for in vivo studies. Future work is necessary to establish an integrated system with comparable release kinetics of iron ions and $\beta$-lap. Second, safety and toxicity of the co-delivered micelle therapeutics will need to be carefully examined in normal tissues and organs, in particular in reticuloendothelial system (RES) where higher uptake of the micelle nanoparticles is anticipated. Our current data show that dicourmarol can effectively block the formation of $\mathrm{H}_{2} \mathrm{O}_{2}$ by $\beta$-lap in NQO1-expressing cancer cells (Fig. 3B), leading to dramatically reduced toxicity from $\beta$-lap-SPION co-treatment. It is shown that normal tissues have low levels of NQO1 and high levels of catalase [51, 52], which should render significantly lowered toxicity from the co-treatment. Further mechanistic correlation of the toxicity and antitumor efficacy of $\beta$-lap-SPION micelles with intended biomarkers (e.g. NQO1 and catalase) will be important to establish the therapeutic window for the combination therapy.

\section{Conclusion}

In summary, we report the use of $\mathrm{pH}$-responsive SPION-micelles to synergize with an anticancer drug, $\beta$-lap, for improved therapeutic efficacy in NQO1-overexpressing cancer cells. SPION-micelles were internalized into acidic organelles, where iron ions were released from micelles in a $\mathrm{pH}$-dependent manner. The iron ions further reacted with $\mathrm{H}_{2} \mathrm{O}_{2}$ generated from $\beta$-lap to produce highly active hydroxyl radicals, escalated ROS stress levels in cancer cells, resulting in catalase-sensitive, NQO1-specific $\beta$-lap drug cytotoxicity. These biocompatible SPION-micelle imaging vehicles display a synergistic efficacy in combination with ROS-generating anticancer agents for cancer therapy and opens up a new avenue for their applications in theranostic nanomedicine.

\section{Acknowledgments}

This work was supported by the National Institutes of Health to JG (R21EB005394, R01CA122994 and R01CA129011) and DAB (R01CA102792).

\section{Competing Interests}

The authors declare no competing financial interest. 


\section{References}

1. Sajja HK, East MP, Mao H, Wang YA, Nie S, Yang L. Development of multifunctional nanoparticles for targeted drug delivery and noninvasive imaging of therapeutic effect. Curr Drug Discovery Technol. 2009; 6: 43-51.

2. Thorek DL, Tsourkas A. Size, charge and concentration dependent uptake of iron oxide particles by non-phagocytic cells. Biomaterials. 2008; 29: 3583-90.

3. Xie J, Liu G, Eden HS, Ai H, Chen X. Surface-engineered magnetic nanoparticle platforms for cancer imaging and therapy. Acc Chem Res. 2011; 44: 883-92.

4. Hao R, Xing R, Xu Z, Hou Y, Gao S, Sun S. Synthesis, functionalization, and biomedical applications of multifunctional magnetic nanoparticles. Adv Mater. 2010; 22: 2729-42.

5. Wang YX, Hussain SM, Krestin GP. Superparamagnetic iron oxide contrast agents: physicochemical characteristics and applications in MR imaging. Eur Radiol. 2001; 11: 2319-31.

6. Sun C, Lee JSH, Zhang M. Magnetic nanoparticles in MR imaging and drug delivery. Adv Drug Deliv Rev. 2008; 60: 1252-65.

7. Shubayev VI, Pisanic Ii TR, Jin S. Magnetic nanoparticles for theragnostics. Adv Drug Deliv Rev. 2009; 61: 467-77.

8. Ai H, Flask C, Weinberg BD, Shuai X, Pagel MD, Farrell D, et al. Magnetite-loaded polymeric micelles as novel magnetic resonance probes. Adv Mater. 2005; 17: 1949-52.

9. Kessinger CW, Khemtong C, Togao O, Takahashi M, Sumer BD, Gao J. In vivo angiogenesis imaging of solid tumors by alpha(v)beta(3)-targeted, dual-modality micellar nanoprobes. Exp Biol Med (Maywood). 2010; 235: 957-65.

10. Kessinger CW, Togao O, Khemtong C, Huang G, Takahashi M, Gao J. Investigation of in vivo targeting kinetics of alpha(v)beta(3)-specific superparamagnetic nanoprobes by time-resolved MRI. Theranostics. 2011; 1: 263-73.

11. Khemtong C, Kessinger CW, Ren JM, Bey EA, Yang SG, Guthi JS, et al. In vivo off-resonance saturation magnetic resonance imaging of alpha(v)beta(3)-targeted superparamagnetic nanoparticles. Cancer Res. 2009; 69: 1651-8.

12. Nasongkla N, Bey E, Ren J, Ai H, Khemtong C, Guthi JS, et al. Multifunctional polymeric micelles as cancer-targeted, MRI-ultrasensitive drug delivery systems. Nano Lett. 2006; 6: 2427-30.

13. Guthi JS, Yang SG, Huang G, Li S, Khemtong C, Kessinger CW, et al. MRI-visible micellar nanomedicine for targeted drug delivery to lung cancer cells. Mol Pharm. 2010; 7: 32-40.

14. Mahmoudi M, Sant S, Wang B, Laurent S, Sen T. Superparamagnetic iron oxide nanoparticles (SPIONs): Development, surface modification and applications in chemotherapy. Adv Drug Deliv Rev. 2011; 63: 24-46.

15. Arbab AS, Wilson LB, Ashari P, Jordan EK, Lewis BK, Frank JA. A model of lysosomal metabolism of dextran coated superparamagnetic iron oxide (SPIO) nanoparticles: implications for cellular magnetic resonance imaging. NMR Biomed. 2005; 18: 383-9.

16. Lin M, Kim HH, Kim H, Muhammed M, Kyung Kim D. Iron oxide-based nanomagnets in nanomedicine: fabrication and applications. Nano Rev. 2010; 1: 4883.

17. Pouliquen D, Lejeune JJ, Perdrisot R, Ermias A, Jallet P. Iron-oxide nanoparticles for use as an MRI contrast agent - pharmacokinetics and metabolism. Magn Reson Imaging. 1991; 9: 275-83.

18. Arbab AS, Bashaw LA, Miller BR, Jordan EK, Lewis BK, Kalish H, et al. Characterization of biophysical and metabolic properties of cells labeled with superparamagnetic iron oxide nanoparticles and transfection agent for cellular MR imaging. Radiology. 2003; 229: 838-46.

19. Gao J, Gu H, Xu B. Multifunctional magnetic nanoparticles: design, synthesis, and biomedical applications. Acc Chem Res. 2009; 42: 1097-107.

20. Skotland T, Sontum PC, Oulie I. In vitro stability analyses as a model for metabolism of ferromagnetic particles (Clariscan(TM)), a contrast agent for magnetic resonance imaging. J Pharm Biomed Anal. 2002; 28: 323-9.

21. Yang CY, Hsiao JK, Tai MF, Chen ST, Cheng HY, Wang JL, et al. Direct labeling of hMSC with SPIO: the long-term influence on toxicity, chondrogenic differentiation capacity, and intracellular distribution. Mol Imaging Biol. 2011; 13: 443-51.

22. Schlorf $\mathrm{T}$, Meincke M, Kossel E, Gluer CC, Jansen O, Mentlein R. Biological properties of iron oxide nanoparticles for cellular and molecular magnetic resonance imaging. Int J Mol Sci. 2010; 12: 12-23.

23. Weissleder R, Stark DD, Engelstad BL, Bacon BR, Compton CC, White DL, et al. Superparamagnetic iron oxide: pharmacokinetics and toxicity. AJR Am J Roentgenol. 1989; 152: 167-73.
24. Kolter T, Sandhoff K. Lysosomal degradation of membrane lipids. FEBS Lett. 2010; 584: 1700-12.

25. Wang J, Pantopoulos K. Regulation of cellular iron metabolism. Biochem J. 2011; 434: 365-81.

26. Goldstein S, Meyerstein D, Czapski G. The Fenton reagents. Free Radic Biol Med. 1993; 15: 435-45.

27. Puzyn T, Rasulev B, Gajewicz A, Hu X, Dasari TP, Michalkova A, et al. Using nano-QSAR to predict the cytotoxicity of metal oxide nanoparticles. Nat Nanotechnol. 2011; 6: 175-8.

28. Brillas E, Sires I, Oturan MA. Electro-Fenton process and related electrochemical technologies based on Fenton's reaction chemistry. Chem Rev. 2009; 109: 6570-631.

29. Voinov MA, Pagan JO, Morrison E, Smirnova TI, Smirnov AI. Surface-mediated production of hydroxyl radicals as a mechanism of iron oxide nanoparticle biotoxicity. J Am Chem Soc. 2010; 133: 35-41.

30. Trachootham D, Alexandre J, Huang P. Targeting cancer cells by ROS-mediated mechanisms: a radical therapeutic approach? Nat Rev Drug Discovery. 2009; 8: 579-91.

31. Cabello CM, Bair WB, 3rd, Wondrak GT. Experimental therapeutics: targeting the redox Achilles heel of cancer. Curr Opin Investig Drugs. 2007; 8: 1022-37.

32. Fry FH, Jacob C. Sensor/effector drug design with potential relevance to cancer. Curr Pharm Des. 2006; 12: 4479-99.

33. Magda D, Miller RA. Motexafin gadolinium: a novel redox active drug for cancer therapy. Semin Cancer Biol. 2006; 16: 466-76.

34. Alexandre J, Nicco C, Chereau C, Laurent A, Weill B, Goldwasser F, et al. Improvement of the therapeutic index of anticancer drugs by the superoxide dismutase mimic mangafodipir. J Natl Cancer Inst. 2006; 98: 236-44.

35. Blanco E, Bey EA, Khemtong C, Yang S-G, Setti-Guthi J, Chen H, et al. $\beta$-Lapachone micellar nanotherapeutics for non-small cell lung cancer therapy. Cancer Res. 2010; 70: 3896-904.

36. Bey EA, Bentle MS, Reinicke KE, Dong Y, Yang C-R, Girard L, et al. An NQO1- and PARP-1-mediated cell death pathway induced in non-small-cell lung cancer cells by $\beta$-lapachone. Proc Natl Acad Sci U S A. $2007 ; 104: 11832-7$

37. Gutteridge JM. Iron promoters of the Fenton reaction and lipid peroxidation can be released from haemoglobin by peroxides. FEBS Lett. 1986; 201: 291-5.

38. Maxfield FR, McGraw TE. Endocytic recycling. Nat Rev Mol Cell Biol. 2004; 5: 121-32.

39. Kon K, Kim JS, Uchiyama A, Jaeschke H, Lemasters JJ. Lysosomal iron mobilization and induction of the mitochondrial permeability transition in acetaminophen-induced toxicity to mouse hepatocytes. Toxicol Sci. 2010; 117: 101-8.

40. Sun SH, Zeng H, Robinson DB, Raoux S, Rice PM, Wang SX, et al. Monodisperse MFe2O4 ( $\mathrm{M}=\mathrm{Fe}, \mathrm{Co}, \mathrm{Mn})$ nanoparticles. J Am Chem Soc. 2004; 126: 273-9.

41. Zhou K, Wang Y, Huang X, Luby-Phelps K, Sumer BD, Gao J. Tunable, ultrasensitive $\mathrm{pH}$-responsive nanoparticles targeting specific endocytic organelles in living cells. Angew Chem -Int Edit. 2011; 50: 6109-14.

42. Pink JJ, Planchon SM, Tagliarino C, Varnes ME, Siegel D, Boothman DA. $\mathrm{NAD}(\mathrm{P}) \mathrm{H}$ :Quinone oxidoreductase activity is the principal determinant of beta-lapachone cytotoxicity. J Biol Chem. 2000; 275: 5416-24.

43. Bae Y, Kataoka K. Intelligent polymeric micelles from functional poly(ethylene glycol)-poly(amino acid) block copolymers. Adv Drug Deliv Rev. 2009; 61: 768-84.

44. Bentle MS, Reinicke KE, Bey EA, Spitz DR, Boothman DA. Calcium-dependent modulation of poly(ADP-ribose) polymerase-1 alters cellular metabolism and DNA repair. J Biol Chem. 2006; 281: 33684-96.

45. Khaket TP, Ahmad R. Biochemical studies on hemoglobin modified with reactive oxygen species (ROS). Appl Biochem Biotech. 2011; 164: 1422-30.

46. Richardson DR, Ponka P. Development of iron chelators to treat iron overload disease and their use as experimental tools to probe intracellular iron metabolism. Am J Hematol. 1998; 58: 299-305.

47. Abu-Murad C, Nordmann R. Effect of two iron-chelators, desferrioxamine and diethylenetriaminepentaacetic acid, on the development of tolerance to the physical dependence on ethanol in mice. Drug Alcohol Depend. 1983; 12: 371-9.

48. Dong Y, Bey EA, Li LS, Kabbani W, Yan J, Xie XJ, et al. Prostate cancer radiosensitization through poly(ADP-Ribose) polymerase-1 hyperactivation. Cancer Res. 2010; 70: 8088-96.

49. Bentle MS, Reinicke KE, Dong Y, Bey EA, Boothman DA. Nonhomologous end joining is essential for cellular resistance to the novel antitumor agent, beta-lapachone. Cancer Res. 2007; 67: 6936-45. 
50. Gao L, Zhuang J, Nie L, Zhang J, Zhang Y, Gu N, et al. Intrinsic peroxidase-like activity of ferromagnetic nanoparticles. Nat Nanotechnol. 2007; 2: 577-83.

51. Hartz JW, Funakoshi S, Deutsch HF. The levels of superoxide dismutase and catalase in human tissues as determined immunochemically. Clin Chim Acta. 1973; 46: 125-32.

52. Radjendirane V, Joseph P, Lee YH, Kimura S, Klein-Szanto AJ, Gonzalez FJ, et al. Disruption of the DT diaphorase (NQO1) gene in mice leads to increased menadione toxicity. J Biol Chem. 1998; 273: 7382-9. 